\title{
TIME EVOLUTION OF THE HALDANE STATE AFTER A QUANTUM QUENCH
}

\author{
Dariusz Woźniak ${ }^{1}$, Andrzej Drzewiński ${ }^{1}$, Grzegorz Kamieniarz ${ }^{2}$ \\ ${ }^{1}$ Institute of Physics, University of Zielona Góra, Zielona Góra, Poland \\ ${ }^{2}$ Faculty of Physics, A. Mickiewicz University, Poznań, Poland \\ ${ }^{1}$ dwozniak.uz@gmail.com, I'a.drzewinski@if.uz.zgora.pl, ${ }^{2}$ gjk@amu.edu.pl
}

\begin{abstract}
In the framework of the Matrix Product States representation the effect of a sudden quench of the uniaxial anisotropy on the time evolution of the Haldane state has been investigated. The existence of the non-vanishing string correlations in the limit of a large distance in the Haldane phase has been verified. The overlap of the initial and time-evolved states, the so-called Loschmidt echo, has been investigated.
\end{abstract}

Keywords: computational statistical mechanics, the ground state and dynamical properties of magnetic chains

\section{Introduction}

Recent research with ultracold atoms in optical lattices has opened the experimental way to investigate the ground state and dynamical properties of magnetic chains [1]. A great deal of interest in one-dimensional $(d=1)$ AF Heisenberg chains has been originated by Haldane's conjecture that $d=1$ quantum Heisenberg antiferromagnets have qualitatively different properties according to whether the spin value is an integer or a half-integer. The elementary excitation spectrum of such a system with integer spin is gapful, whereas for the system with a half-integer spin is gapless [2].

The $\mathrm{S}=1$ antiferromagnetic Heisenberg chain is governed by the following Hamiltonian:

$$
\hat{H}_{J}=J \sum_{i=1}^{N-1} \hat{S}_{i}^{x} \hat{S}_{i+1}^{x}+\hat{S}_{i}^{y} \hat{S}_{i+1}^{y}+\hat{S}_{i}^{z} \hat{S}_{i+1}^{z}
$$

where the parameter $J$ is an exchange coupling constant. In order to study the ground-state magnetization dynamics we have considered the chain with $N=102$ sites and open boundary conditions.

It is known that to construct the ground state of the Hamiltonian (1) each original $\mathrm{S}=1$ spin can be written as two $\mathrm{S}=1 / 2$ spins in a triplet state. Based on them the ground state, the so-called, valence-bond-state can be built $[3,4]$. However, when we are dealing with an open chain, two effective free $S=1 / 2$ spins appear 
at the ends and the ground state is split into four states converging to the same ground state as the length of the chain tends to infinity [3]. But when both $\mathrm{S}=1$ spins at the ends are replaced by the $S=1 / 2$ spins a nonzero energy gap between the singlet ground state and the first excited state is restored. For this reason, such an approach was adopted in the current paper.

Besides exhibiting the famed Haldane gap, the $\mathrm{S}=1$ AF Heisenberg chain has been found to have other surprising features. First, due to the existence of a finite energy gap above the ground state, the spin-spin correlations should decay exponentially. Second, there is a form of non-local hidden order, the antiferromagnetic alignment of \pm 1 spins after omitting all the sites with spin projection 0 . It leads to the existence of the string order parameter $O^{\alpha}(\alpha=x, y, z)$ that should be nonzero in the Haldane phase [5]. It is defined by the string correlation functions $O_{j, k}^{\alpha}$ in the following way:

$$
O^{\alpha}:=\lim _{|j-k| \rightarrow \infty}\left\langle\hat{O}_{j, k}^{\alpha}\right\rangle \neq 0,
$$

where $\hat{O}_{j, k}^{\alpha}:=\hat{S}_{j}^{\alpha} \otimes \exp \left(i \pi \hat{S}_{j+1}^{\alpha}\right) \otimes \cdots \otimes \exp \left(i \pi \hat{S}_{k-1}^{\alpha}\right) \otimes \hat{S}_{k}^{\alpha}$ and $\hbar \hat{S}_{j}^{\alpha}$ is the $\alpha$-th component of the spin operator at the $j$-th site.

In real systems, the magnetic uniaxial anisotropy can be created through the environment which implies adding the additional term

$$
\hat{H}_{D}=D \sum_{i=1}^{N}\left(S_{i}^{z}\right)^{2}
$$

where $D$ is the uniaxial anisotropy parameter. Depending on the sign the parameter $D$ describes the system with an easy axis (negative $D$ ) or an easy plane (positive $D$ ). When the uniaxial anisotropy is taken into account, the Haldane phase was found to exist between $D \sim-0.2$ and $D \sim 1$ [6].

In order to investigate the ground state and its dynamical properties after a sudden quantum quench, the Matrix Product States (MPS) formalism has been employed $[7,8]$. The MPS formalism is especially appropriate for the study of gapped systems. Assume that the initial quantum state $\left|\psi_{0}\right\rangle$ is the ground state of the Hamiltonian $H_{J}$. For $t>0$ the time evolution is governed by the anisotropic Hamiltonian $H=H_{J}+H_{D}$ and then the initial quantum state $\left|\psi_{0}\right\rangle$ is never more its eigenstate. As a result, $\left|\psi_{0}\right\rangle$ evolves into a state

$$
|\psi(t)\rangle=\exp (-i \hat{H} t)\left|\psi_{0}\right\rangle
$$

In order to measure the overlap of the time-evolved state and the initial state we can use the so-called Loschmidt echo defined as [8]

$$
L(t)=\left|\left\langle\psi_{0}|\exp (-i \hat{H} t)| \psi_{0}\right\rangle\right|^{2} .
$$


Our goal is to check if there is a relationship between the Haldane gap and relaxation dynamics of the perturbed state. A similar problem was studied by Mazza et al. for an Ising-like anisotropy [9]. Our calculations were carried out for four values of the parameter $D=-1.0,-0.1,0.5,1.0$.

\section{Time evolution of Matrix Product States}

The observation that for physical systems only a minor part of the Hilbert space is involved [10,11], resulting in the rapid development of numerical methods based on a variational method within the space of Matrix Product States (MPS). It corresponds to assigning a finite entanglement content to spins in the ground state. Therefore, any state of the spin chain can be presented in the MPS representation

$$
|\psi\rangle=\sum_{\sigma_{1}, \ldots, \sigma_{N}}^{d_{1}, \ldots, d_{N}} \sum_{a_{1}, \ldots, a_{N-1}}^{D_{1}, \ldots, D_{N-1}} M_{1, a_{1}}^{\sigma_{1}} M_{a_{1}, a_{2}}^{\sigma_{2}} \cdots M_{a_{N-1}, 1}^{\sigma_{N}}|\boldsymbol{\sigma}\rangle
$$

where $|\sigma\rangle=\left|\sigma_{1}, \ldots, \sigma_{N}\right\rangle, d_{i}$ is the dimension of the local base $\left\{\sigma_{i}\right\}$ at the $i$-th site. The matrices $M$ are of dimension $D_{i} \times D_{i}$. Quantity $D_{i}$, called the local bond dimension, is related to the entanglement of neighboring spins. In analogous manner any operator can be written as a Matrix Product Operator (MPO)

$$
\hat{O}=\sum_{\sigma_{1}, \ldots, \sigma_{N}}^{d_{1}, \ldots, d_{N}} \sum_{\sigma_{1}^{\prime}, \ldots, \sigma_{N}^{\prime}}^{d_{1}, \ldots, d_{N}} \sum_{b_{1}, \ldots, b_{N-1}}^{B_{1}, \ldots, B_{N-1}} W_{1, b_{1}}^{\sigma_{1}, \sigma_{1}^{\prime}} W_{b_{1}, b_{2}}^{\sigma_{2}, \sigma_{2}^{\prime}} \cdots W_{b_{N-1}, 1}^{\sigma_{N}, \sigma_{N}^{\prime}}|\boldsymbol{\sigma}\rangle\left\langle\boldsymbol{\sigma}^{\prime}\right| .
$$

Due to the above representation the state space grows only polynomially in the system size (not exponentially as usual). Thus, for $d=1$ strongly correlated systems the time of calculations is significantly reduced. When the variational principle is applied, the ground state can be found by the minimization procedure $\left\langle\psi_{0}\left|H_{J}\right| \psi_{0}\right\rangle$ under the constrain $\left\langle\psi_{0} \mid \psi_{0}\right\rangle=1$ [12]. Moreover, it is one of the most attractive features of the MPS representation that the time evolution can also be performed very efficiently. Therefore, discrete time as $t=N \Delta t$ can be used for the anisotropic Hamiltonian (1), when a second-order Trotter decomposition is applied [10], the time-evolution operator can be presented as:

$$
\exp (-i \hat{H} \Delta t)=\exp \left(-i \hat{H}_{o} \Delta t / 2\right) \exp \left(-i \hat{H}_{e} \Delta t\right) \exp \left(-i \hat{H}_{o} \Delta t / 2\right)+O\left(\Delta t^{3}\right)
$$

where

$$
\begin{gathered}
\hat{H}_{o}=J \sum_{i=1}^{N / 2} \hat{S}_{2 i-1}^{x} \hat{S}_{2 i}^{x}+\hat{S}_{2 i-1}^{y} \hat{S}_{2 i}^{y}+\hat{S}_{2 i-1}^{z} \hat{S}_{2 i}^{z} \\
\hat{H}_{e}=J \sum_{i=1}^{N / 2-1} S_{2 i}^{x} S_{2 i+1}^{x}+S_{2 i}^{y} S_{2 i+1}^{y}+S_{2 i}^{z} S_{2 i+1}^{z}+D \sum_{i=2}^{N-1}\left(S_{i}^{z}\right)^{2} .
\end{gathered}
$$


Then the step of the time-evolution algorithm takes a very simple form [10]: one starts from $|\psi(t)\rangle$ and performs the following steps:

1. Applying the MPO of the odd bonds to $|\psi(t)\rangle$

2. Applying the MPO of the even bonds to $\exp \left(-i H_{0} \Delta t / 2\right)|\psi(t)\rangle$

3. Applying the MPO of the odd bonds to $\exp \left(-i H_{\mathrm{e}} \Delta t\right) \exp \left(-i H_{0} \Delta t / 2\right)|\psi(t)\rangle$

4. Compressing the MPO $\exp \left(-i H_{0} \Delta t / 2\right) \exp \left(-i H_{\mathrm{e}} \Delta t\right) \exp \left(-i H_{0} \Delta t / 2\right)|\psi(t)\rangle$ to the starting dimension as $|\psi(t+\Delta t)\rangle$.

In the present studies the maximal local bond dimension was $D_{i}=80$, whereas the time step was $\Delta t=10^{-4}$.

\section{Results}

First, we have examined the established ground state of (1) in terms of features typical for the Haldane system. Since the Hamiltonian (1) is isotropic in the spin space, the components of the spin-spin correlation functions and of the string correlation functions are all equal, respectively. Moreover, all correlated spins are separated by a distance $2 k-1$ arranged symmetrically with respect to the center of the chain.

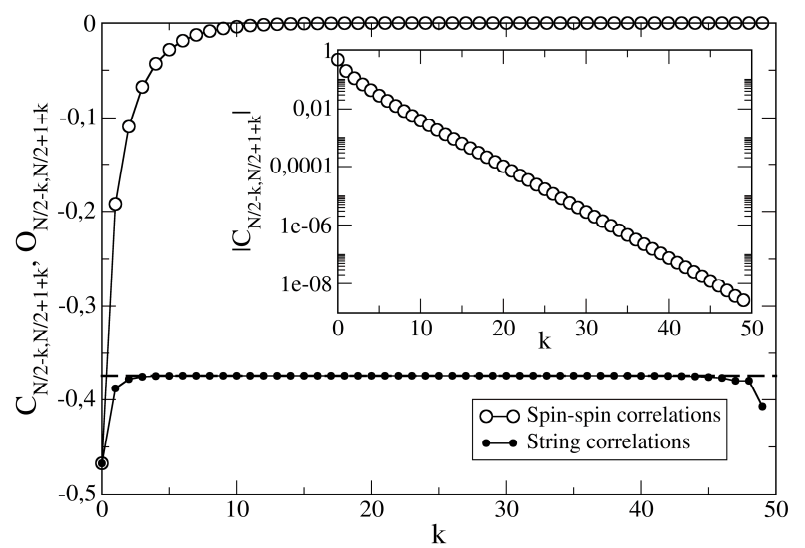

Fig. 1. The spin-spin and string correlation functions. The inset presents a linear-log plot of the spin-spin correlation function. The dashed line shows the value $O^{\alpha} \sim-0.3743$

As one can see in Figure 1, the spin-spin correlation functions decay exponentially as could be expected. Next, the string correlation functions show a wide plateau followed by a gradual decline. The decline is due to the influence of the chain ends when the spin-spin distance reaches a maximum value. So, when the distance between the spins increases the value of the string correlation tends towards a constant value $O^{\alpha} \sim-0.3743$ [13] which indicates the non-local string long-range ordering.

Next we present numerical studies of the quench dynamics of the $\mathrm{S}=1 \mathrm{AF}$ Heisenberg chain after a rapid change of the environmental anisotropy. As shown 
in Figure 2, the Loschmidt echo behavior significantly depends on the anisotropy parameter. When the absolute value $D$ is high and corresponds to the parameter range where the Haldane gap is closed [6], the Loschmidt echo decays exponentially. For the other cases the Loschmidt echo exhibits an oscillation superimposed on the decreasing function going to a finite value. If the parameter $D$ is negative, this limiting value of the Loschmidt echo is clearly higher than for positive values of $D$.

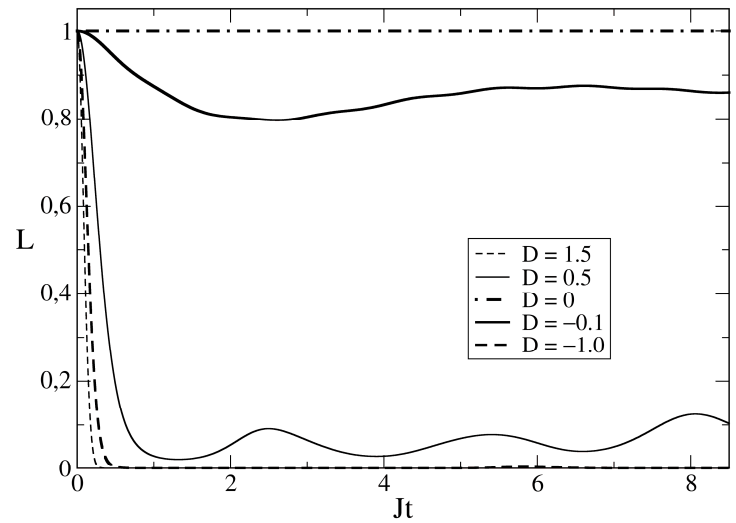

Fig. 2. The time evolution of the Loschmidt echo. The case $D=0$ is the reference case without a quantum quench where the overlap between initial state and the evolved state is perfect

\section{Conclusions}

We have examined the ground-state response of a finite $\mathrm{S}=1$ antiferromagnetic Heisenberg chain after a sudden change of the environmental uniaxial anisotropy. As we have checked, the initial state was taken to be the pure Haldane state. After quenching, the system evolved under the influence of a changed environment (within or outside of the Haldane phase regime).

The Loschmidt echo allows one to quantify the sensitivity of quantum evolution to perturbations. When the value $D$ corresponds to the regime where the Haldane gap is closed, the Loschmidt echo decays exponentially. Otherwise the Loschmidt echo exhibits an oscillation superimposed on the decreasing function going to a finite value. Moreover, it turned out that the initial Haldane state is disturbed more by the easy-plane anisotropy than by the easy-axis one.

\section{Acknowledgments}

D.W. acknowledges support as a scholar within Submeasure 8.2.2 Regional Innovation Strategies, Measure 8.2 Transfer of knowledge, Priority VIII Regional human resources for the economy Human Capital Operational Programme co-financed by European Social Fund and state budget. 


\section{References}

[1] Bloch I., Dalibard J., Zwerger W., Many-body physics with ultracold gases, Rev. Mod. Phys. 2008, 80, 885- 964; Simon J., Bakr W.S., Ma R., Tai M.E., Preiss P.M., Greiner M., Quantum simulation of antiferromagnetic spin chains in an optical lattice, Nature 2011, 472, 307-312.

[2] Haldane F.D.M., Nonlinear field theory of large-spin Heisenberg antiferromagnets: semiclassically quantized solitons of the one-dimensional easy-axis Néel state, Phys. Rev. Lett. 1983, 50, 1153-1156; Haldane F.D.M., Continuum dynamics of the 1-D Heisenberg antiferromagnet: Identification with the O(3) nonlinear sigma model, Phys. Lett. A 1983, 93, 464-468.

[3] Affleck I., Kennedy T., Lieb E.H., Tasaki H., Rigorous results on valence-bond ground states in antiferromagnets, Phys. Rev. Lett. 1987, 59, 799-802.

[4] White S.R., Huse D.A., Numerical renormalization-group study of low-lying eigenstates of the antiferromagnetic $S=1$ Heisenberg chain, Phys. Rev. B 1993, 48, 3844-3852.

[5] den Nijs M., Rommelse K., Preroughening transitions in crystal surfaces and valence-bond phases in quantum spin chains, Phys. Rev. B 1989, 40, 4709-4734.

[6] Chen W., Hida K., Sanctuary B.C., Ground-state phase diagram of $S=1$ XXZ chains with uniaxial single-ion-type anisotropy, Phys. Rev. B 2003, 67, 104401.

[7] Östlund S., Rommer S., Thermodynamic limit of density matrix renormalization, Phys. Rev. Lett. 1995, 75, 3537-3540.

[8] Pozsgay B., The dynamical free energy and the Loschmidt echo for a class of quantum quenches in the Heisenberg spin chain, J. Stat. Mech. 2013, 13, 10028.

[9] Mazza L., Rossini D., Endres M., Fazio R., Out-of-equilibrium dynamics and thermalization of string order, Phys. Rev. B 2014, 90, 020301.

[10] Schollwöck U., The density-matrix renormalization group, Rev. Mod. Phys. 2005, 77, 259-315; Schollwöck U., The density-matrix renormalization group in the age of matrix product states, Annals of Physics 2011, 326, 96-192.

[11] Verstraete F., Cirac K., Matrix product states represent ground states faithfully, Phys. Rev. B 2006, 73, 094423; Verstraete F., Murg V., Cirac K., Matrix product states, projected entangled pair states, and variational renormalization group methods for quantum spin systems, Adv. Phys. 2008, 57, 143-224.

[12] Woźniak D., Drzewiński A., Kamieniarz G., Matrix-product states for the Ising model in a transverse field, Acta Physicae Superficierum 2012, 12, 187-194.

[13] Alcaraz F.C., Hatsugai Y., String correlation functions in the anisotropic spin-1 Heisenberg chain, Phys. Rev. B 1992. 